\title{
Selective gene expression profiling of mTOR-associated tumor suppressor and oncogenes in ovarian cancer
}

\author{
Piotr Laudański ${ }^{1}$, Oksana Kowalczuk ${ }^{2}$, Dagmara Klasa-Mazurkiewicz ${ }^{3}$, \\ Tomasz Milczek ${ }^{4}$, Dominik Rysak-Luberowicz ${ }^{5}$, Magdalena Garbowicz ${ }^{6}$, \\ Włodzimierz Baranowski ${ }^{5}$, Radosław Charkiewicz ${ }^{2}$, Jacek Szamatowicz ${ }^{7}$, \\ Lech Chyczewski ${ }^{8}$
}

\author{
${ }^{1}$ Department of Perinatology, Medical University of Bialystok, Poland \\ ${ }^{2}$ Department of Clinical Molecular Biology, Medical University of Bialystok, Poland \\ ${ }^{3}$ Department of Perinatology, Medical University of Gdansk, Poland \\ ${ }^{4}$ Department of Gynecologic Oncology, Medical University of Gdansk, Poland \\ ${ }^{5}$ Department of Gynecology and Gynecologic Oncology, Military Institute \\ of Health Sciences, Warsaw, Poland \\ ${ }^{6}$ Department of Thoracic Surgery, Medical University of Bialystok, Poland \\ ${ }^{7}$ Department of Gynecology, Medical University of Bialystok, Poland \\ ${ }^{8}$ Department of Medical Pathomorphology, Medical University of Bialystok, Poland
}

\begin{abstract}
The aim of this study was to selectively profile the activation status of mammalian target of rapamycin (mTOR)-associated oncogenes and tumor suppressor genes (TSGs) in ovarian cancer specimens, healthy ovaries and benign ovarian tumors, including endometrial cysts. We used a novel type of microfluidic gene array to examine the expression of 15 human tumor suppressors and oncogenes in ovarian cancer specimens of 53 patients, benign ovarian cysts of 29 women (endometrial and simple) and 11 healthy ovaries of individuals in whom the material was obtained during total hysterectomies performed because of fibroid changes. The array was custom-designed to include the following genes: NF1, RHEB, mTOR1, AKT-1, PTEN, TSC1, TSC2, KRAS, RPS6KB1, 4EBP1, TP53, EIF4E, STK11, PIK3CA and BECN1. Confirmatory immunohistochemical detection was performed for a group of selected proteins. Particularly significant differences were observed as to the expression of PTEN ( $\mathrm{p}<0.0001)$, TP53 ( $\mathrm{p}=0.0003)$, PIK3CA $(\mathrm{p}=0.0003)$ and $B E C N 1(\mathrm{p}=0.0014)$ which were shown to be downregulated in cancer patients when compared to healthy ovaries and benign ovarian cysts (endometrial and simple). These markers did not show association with grade or stage of the tumor. Immunohistochemistry showed that PTEN, TP53, PIK3CA and BECN1 proteins are expressed in ovarian cancer. Our results indicate that there are significant differences in the expression of some of the mTOR-related tumor suppressors and oncogenes which could be associated with the pathogenesis of ovarian cancer. (Folia Histochemica et Cytobiologica 2011; Vol. 49, No. 2, pp. 317-324)
\end{abstract}

Key words: ovarian cancer, mTOR pathway, TP53, PIK3CA, PTEN, BECN1

Correspondence address: P. Laudański, Department of Perinatology, Medical University of Bialystok, Marii Sklodowskiej-Curie Str. 24a Bialystok, Poland; tel.: (+ 48) 608344 788, fax: (+ 48 85) 7468605 ;

e-mail:plauda@umwb.edu.pl

\section{Introduction}

Ovarian cancer remains a highly lethal disease which in developed countries accounts for more deaths than all other gynecological malignancies combined: an 
estimated 22,430 new cases and 15,280 deaths occurred in 2007 in the USA alone [1].

The molecular pathology of ovarian carcinomas is heterogeneous and involves various putative precursor lesions and multiple pathways of development. Although very little is known regarding the molecular mechanisms involved in the biochemical alterations of cell signaling, most of them lead to abnormal growth which depends on altered stimulation by growth factor receptor-cell signaling pathways. Stimuli follow different pathways, but a substantial number of them converge in the mammalian target of rapamycin (mTOR) which plays a central role in cancerogenesis and, as we have recently shown, its key pathway components have been implicated in the development of endometriosis $[2,3]$.

mTOR is a conserved seronine/threonine kinase which functions by integrating extracellular signals (growth factors and hormones). mTOR enhances translation initiation in part by phosphorylating a group of its major targets, such as: transcription factor eIF4E (mRNA cap-binding protein) which is a known potent oncogene, and selected tumor suppressor genes, like: $4 E-B P 1$ (the so called inhibitory 4E-binding protein) considered a hallmark of cell signaling in ovarian cancer [4], and ribosomal protein S6 kinases (e.g. oncogene S6K1) that co-operate to regulate gene expression and translation processes [2].

S6K1 has also been shown to repress the phosphatidylinositol 3-kinase (PI3K) related pathways. $P I 3 K$ oncogene is a heterodimer that is composed of a p85 regulatory and a p110 catalytic subunit (coded for by the PIK3CA), and its aberrantly high activity has been implicated in cell transformation and tumor progression in a variety of human cancers [5]. PI3K converts PIP2 to PIP3 (phosphatidylinositol-3, 4,5-triphosphate), which is actively opposed by its phosphatase PTEN (phosphatase and tensin homolog deleted on chromosome ten) tumor suppressor of which loss of function has been found in many advanced human cancers [6]. PIP3 recruits one of the most potent oncogenes $A K T$ [5] to the membrane where it is activated by phosphorylation. Once activated, AKT phosphorylates a multitude of proteins, with the net result of increased cellular survival, proliferation, growth and metabolism. Specifically, phosphorylation of mTOR by AKT occurs through inactivation of the tuberous sclerosis complex - heterodimer consisting of unphosphorylated tumor suppressors TSC1 and TSC2 [7]. The complex acts as a GTPase-activating protein (GAP), inhibiting the small G-protein Rheb (Ras homolog enriched in brain), an oncogene of which expression is elevated in many tumor cells [8].

TSC1, TSC2 and Rheb also contribute to the activation of mTOR after losing such tumor suppressors as: NF1 (neurofibromatosis 1), LKB1 (also known as serine-threonine kinase 11) or p53 [2]. NF1 also encodes a GAP for Rat Sarcoma (RAS), of which mutations result in hyperactivation of the protein and are among the most frequent alterations in human cancers. It has also been found that prolonged activation of $k$-ras can occur in tumors by mechanisms that do not involve mutations [9].

$P 53$, which plays a pivotal role in safeguarding the integrity of the genome, harbors mutations in more than $50 \%$ of human cancers of all tissue origins [10] and is also a central signal integrator of stress, such as DNA damage or hypoxia. When activated, it initiates cellular tumor suppression programs such as apoptosis, cell cycle arrest or autophagy.

Autophagy was recently established as a novel tumor suppression mechanism which leads to lysosomal degradation of cytoplasmic components. Genetic evidence shows that compromised autophagy plays a causal role in tumorigenesis. Specifically, decreased expression of BECN1 (beclin-1) is associated with human breast cancer, ovarian and prostate cancer [11]. Moreover, autophagy is negatively regulated by mTOR which is a central protein that integrates signals from nutrients and growth factors [12].

Our aim was to profile the expression status of the aforementioned key tumor suppressor genes and oncogenes that could be functionally related to the mTOR pathway [13] in ovarian cancer. We also attempted to show that the low-density microfluidic gene chip is an effective technique to examine the expression of different genes in such a heterogeneous tissue as the ovary.

\section{Material and methods}

Tissue samples $(\mathrm{n}=93)$ from patients with invasive ovarian cancer, benign ovarian cysts and normal ovaries were collected during surgery performed in the Department of Gynecology of the Medical University of Bialystok, the Department of Gynecologic Oncology of the Medical University of Gdansk, and the Department of Gynecology and Gynecologic Oncology of the Military Institute of Health Sciences in Warsaw. The operations were performed in 2006 and 2009. We initially collected samples from a total of 80 patients diagnosed with an ovarian malignancy $(n=53)$ on frozen section who underwent full surgical staging or tumor debulking as clinically indicated. A piece of each collected tissue was divided into two halves and placed separately in buffered formalin for histopathological studies and RNA later (Sigma Aldrich, Poland) for molecular analysis. The latter was stored for 24 hours at $4^{\circ} \mathrm{C}$ and then tissues were transferred and stored at $-80^{\circ} \mathrm{C}$. Two opposite margins were checked by means of histology in all cases and proven to consist of cancer tissue only (>90\%). Benign 
ovarian cysts (endometrial and simple), initially collected during laparoscopy from 45 patients, as well as normal ovaries removed from 19 patients during total hysterectomy performed because of fibroids during laparotomy, were chosen as controls. The study was approved by the Institutional Review Board (IRB) and the patients gave their informed consent before inclusion in the study.

The RNA isolation was performed using a total RNA isolation kit from Macherey-Nagel (Duren, Germany) according to the manufacturer's protocol. RNA concentration and quality was determined using a NanoDrop spectrophotometer (Kisker, Steinfurt, Germany) and the Agilent Bioanalyzer 2100 (Agilent Technologies, Perlan, Poland).

Total RNA was subsequently converted to single stranded cDNA and loaded into the custom-designed 16 gene expression assay (three replicates per assay) of the TaqMan real-time PCR microfluidic gene array system (Applied Biosystems, Warsaw, Poland). The assay Ids, including house-keeping gene, and detailed procedures (including thermal cycle PCR conditions) were exactly the same as those described in our most recent publication [3].

Gene expression values (RQ-relative quantity) were calculated based on the $\Delta \Delta \mathrm{C}_{\mathrm{t}}$ method, where one sample was designated as the calibrator, through which all other samples were analyzed [14].

Immunohistochemistry. For the immunohistochemical studies, a representative section from the resected specimen was selected. Four-micrometer-thick sections were deparaffinized and dehydratated. Endogenous peroxidase activity was blocked by Peroxidase Blocking Reagent for five minutes at room temperature. For antigenic recovery, the material was immersed in citrate buffer $(\mathrm{pH}=6)$ and placed in a microwave oven for 20 minutes. After rinsing in PBS buffer $\mathrm{pH}=7.4$ (P53, BECN1) and Tris (PTEN, PIK3CA), the sections were incubated with studied primary antibodies for 24 hours at $4^{\circ} \mathrm{C}$, or ten minutes at room temperature. PTEN (anti-human/mouse PTEN), p53 (mouse monoclonal antibody), PIK3CA (rabbit anti-PIK 3CA) and BECN1 (mouse monoclonal antibody) were assessed using monoclonal mouse antibodies, namely: anti-human PTEN from R\&D Systems (clone 217702) which was diluted 1:40 in Tris buffer $\mathrm{pH}=7.4$; p53 (Santa Cruz Biotechnology, Santa Cruz, CA, USA, clone BP53.12) diluted 1:100 in blocking serum, PIK3CA (Sigma, HPA009985) diluted 1:50 in Tris buffer pH7.4 and BECN1 (Santa Cruz Biotechnology, clone BP53.12) diluted 1:100 in blocking serum.

Sections were then incubated with biotinylated secondary antibody at room temperature for 30 minutes. Afterwards, sections were incubated with ABC solution (Santa Cruz Biotechnology, BECN, P53 and PIK3CA) and R\&D Systems Cell and Tissue Staining Kits (PTEN), at room temperature for 30 minutes, followed by diaminobenzidine tetrachloride solution at room temperature for ten minutes, and counterstained in Mayer's hematoxylin. Sections were subsequently dehydrated, transferred to xylene and eventually mounted in a resinous mountant. The expression of studied proteins was evaluated with the use of light microscopy (Olympus CX21). Appropriate immunohistochemical controls, positive and negative, were carried out.

For statistical analysis, the Kruskal-Wallis nonparametric test was used to compare RQ values of three groups together (ovarian cancer vs. benign ovarian cysts vs. healthy ovaries). Wilcoxon rank-sum test was then used for the comparison of RQ values between ovarian cancer vs. benign ovarian cysts, ovarian cancer vs. healthy ovaries and benign ovarian cysts vs. healthy ovaries. The significance level was equal to 0.05 . The statistical analysis was performed using a SAS STAT package.

\section{Results}

Patient clinico-pathological characteristics are presented in Table 1. Detailed RQ and p values in three main study groups are presented in Table 2 . Because our control group consisted of two relatively different subgroups of tissues (normal ovaries and benign cysts), we decided to divide our study group into three groups and then perform statistical analysis on them. We succeeded in obtaining adequate RNA only from 53 patients with ovarian cancer, 29 with benign ovarian cysts and 11 healthy ovaries. These comprised our

Table 1. Patient clinico-pathological characteristics

\begin{tabular}{|l|c|c|}
\hline Characteristics & Number & $\%$ \\
\hline Number of evaluated patients & 93 & 100 \\
\hline Age (years) & 49 & \\
Median & $21-86$ & \\
Range & & \\
\hline Types of tissues & 11 & 11.8 \\
Normal ovaries & 29 & 31.1 \\
Benign ovarian cysts & 19 & 20.4 \\
Endometriomas & 10 & 10.7 \\
$\quad$ Simple & 53 & 56.9 \\
\hline Malignant & & \\
\hline Stage of disease & 8 & 15.09 \\
I & 5 & 9.4 \\
II & 31 & 58.4 \\
III & 9 & 16.9 \\
IV & & \\
\hline Histological type & 34 & 64.1 \\
Serous & 8 & 15 \\
Endometrioid & 5 & 9.4 \\
Clear cell & 4 & 7,5 \\
Mucinous & 2 & 3,7 \\
Mixed & 10 & 18.8 \\
\hline Grade & 25 & 33.9 \\
G1 & & \\
G2 & & \\
G3 & & \\
\hline
\end{tabular}


main study group and were used for further molecular analysis.

By the use of Kruskal-Wallis, we could compare three groups together. We found significant differences for most of the studied genes, except for mTOR ( $1=0.86), K R A S(\mathrm{p}=0.59)$ and AKT1 $(\mathrm{p}=0.16)$. Then we attempted to compare, by the use of Wilcoxon rank-sum test, whether differences existed as to the RQ values between combinations of subgroups, i.e. ovarian cancer vs. healthy ovaries, ovarian cancer vs. benign cysts and healthy ovaries vs. benign cysts. We found that only for four genes, namely PTEN, TP53, PIK3CA and BECN1, were there significant differences between ovarian cancer and each of the two control groups (Table 2). The median RQ values of the four genes were significantly lower in patients with ovarian cancer compared to two control groups. No differences were found between normal ovaries and benign cysts for three of the four genes i.e. TP53 ( $\mathrm{p}=0.55)$, PIK3CA $(\mathrm{p}=0.98)$ and $B E C N 1(\mathrm{p}=0.65)$. The only exception, PTEN, exerted the highest median gene expression in normal ovaries which was significantly higher than in benign cysts $(\mathrm{p}=0.003)$ and ovarian cancer subgroup $(\mathrm{p}=0.0019)$, which showed the lowest median concentration. Analysis carried out with a group of malignant tumors $(\mathrm{n}=53)$ showed no statistically significant correlation between all 15 tested genes and age, grade (I, II vs. III) or clinical stage (I, II vs. III, IV).

We also decided to study differences in the median RQ values of all genes between two histological types i.e. serous and endometrial. The number of other types was too small for statistical analysis. We found that the differences were significant for two out of 15 studied genes, namely BECN1 $(\mathrm{p}=0.008)$ and TSC2 $(p=0.034)$. The expression of both genes was decreased in serous ovarian cancer: respective median (minimum-maximum) RQ values for BECN1 and TSC2 were: $0.36(0.09-6.56)$ and $0.32(0.08-15.5)$ compared to endometrioid type: respectively 0.77 (0.4$-9.8)$ and 0.73 (0.46-2.89).

\section{Localization of PTEN, TP53, PIK3CA and $B E C N 1$ in ovarian cancer}

In order to confirm protein expression of genes that were differentially regulated in our study, we studied their immunolocalization in selected cases of four main histological types (serous, endometrioid, clear cell and mucinous) of ovarian cancer. Among studied tissue sections, immunoreactivity for PTEN (nuclear and cytoplasmic) was observed only in clear cell (15\% of cells) and mucinous types in about $5 \%$ (Fig- ures $1 \mathrm{~A}$ and $1 \mathrm{~B}$, respectively) whereas neither expression was observed in studied serous nor endometrioid slides.

TP53 protein localization was nuclear and we could observe strong reaction in 50-60\% of endometrioid (Figure 1C) and 80-90\% of clear cell types (Figure 1D). Very little or no reaction was observed in the serous or mucinous types studied.

PIK3CA exhibited very strong immunohistochemical reaction in all histological types studied and average staining was about $80 \%$ in serous, endometrioid and mucinous types (Figures 1E, 1F, 1G, respectively), whereas we could observe that only $30 \%$ of clear cell cancer cells showed, typical for all types, cytoplasmic reaction (Figure 1H).

In the case of BECN1, we observed a weak cytoplasmic reaction in virtually all histological types studied. In the cases of serous cancer sections, the positive reaction was observed in only $5 \%$ of cells (Figure 1I). Endometrioid cancer cells exhibited 10$-12 \%$ positive reactions (Figure 1J). Despite the fact that we did not observe a positive BECN1 reaction in clear cell or mucinous cancer cells, in every histological type we could see characteristic vascular, specifically endothelial, positive reactions of this protein (see arrow in Figure 1I).

\section{Discussion}

A decrease in PTEN expression, which may lead to loss of its function, is more frequent than can be explained by structural genomic changes alone, since epigenetic phenomena, such as hypermethylation of DNA, represent major restrictions to the transcriptional process. PTEN expression can be lost or greatly reduced in some tumors without any mutation in the coding sequence of the gene in cancers of breast, prostate, non-small cell lung, as well as ovarian cancer [15].

Schondorf et al. showed that expression of PTEN is not altered in the progression of ovarian carcinoma, which suggests that in ovarian cancer PTEN does not play a major role in disease progression [16]. Another study found that the level of PTEN mRNA expression was negatively correlated with clinicopathological staging of ovarian cancer, whereas it was positively correlated with histological differentiation. It has also been shown that mRNA expression level of PTEN gene in ovarian endometrioid cancer was significantly lower than that in ovarian serous cancer [17]. In our study, we were unable to show differences as to the expression of PTENbetween those two histological types of cancer. This was possibly due to an insufficient number of samples (eight endometrioid vs. 34 serous). 
Table 2. Detailed RQ and p values in three main study groups

\begin{tabular}{|c|c|c|c|c|}
\hline Gene & $\begin{array}{l}\text { Ovarian cancer vs. } \\
\text { normal ovaries } \\
\text { vs. benign } \\
\text { ovarian cysts }\end{array}$ & $\begin{array}{l}\text { Ovarian cancer } \\
\quad(n=53) \\
\text { vs. normal ovaries } \\
\quad(n=11)\end{array}$ & $\begin{array}{l}\text { Ovarian cancer } \\
\text { vs. benign ovarian } \\
\text { cysts }(\mathrm{n}=29)\end{array}$ & $\begin{array}{l}\text { Normal ovaries } \\
\text { vs. benign } \\
\text { ovarian cysts }\end{array}$ \\
\hline$N F 1$ & $0.0005^{*}$ & $\begin{array}{c}0.294(0.009-21.780) \\
\text { vs. } 0.614(0.145-3.105)^{* *} \\
0.28^{* * *}\end{array}$ & $\begin{array}{c}0.294(0.009-21.780) \\
\text { vs. } 3.772(0.079-35.548) \\
0.0003\end{array}$ & $\begin{array}{c}0.614(0.145-3.105) \\
\text { vs. } 3.772(0.079-35.548) \\
0.04\end{array}$ \\
\hline RHEB & 0.0006 & $\begin{array}{c}0.923(0.115-1027) \\
\text { vs. } 0.583(0.382-2.233) \\
0.37\end{array}$ & $\begin{array}{c}0.923(0.115-1027) \\
\text { vs. } 5.134(0.320-22.027) \\
0.003\end{array}$ & $\begin{array}{c}0.583(0.382-2.233) \\
\text { vs. } 5.134(0.320-22.027) \\
0.0007\end{array}$ \\
\hline mTOR & 0.86 & $\begin{array}{c}1.117(0.179-100.386) \\
\text { vs. } 1.060(0.629-2.853) \\
0.8\end{array}$ & $\begin{array}{c}1.117(0.179-100.386) \\
\text { vs. } 1.296(0.195-7.809) \\
0.62\end{array}$ & $\begin{array}{c}1.060(0.629-2.853) \\
\text { vs. } 1.296(0.195-7.809) \\
0.81\end{array}$ \\
\hline PTEN & 0.0003 & $\begin{array}{c}0.164(0.005-2549) \\
\text { vs. } 8.18115(0.511-14.965) \\
0.0019\end{array}$ & $\begin{array}{c}0.164(0.005-2549) \\
\text { vs. } 0.938(0.062-7.380) \\
0.017\end{array}$ & $\begin{array}{c}8.181(0.511-14.965) \\
\text { vs. } 0.938(0.062-7.380) \\
0.003\end{array}$ \\
\hline$T S C 1$ & 0.0005 & $\begin{array}{c}0.532(0.010-53.708) \\
\text { vs. } 1.153(0.272-5.069) \\
0.11\end{array}$ & $\begin{array}{c}0.532(0.010-53.708) \\
\text { vs. } 5.182(0.044-102.491) \\
0.0004\end{array}$ & $\begin{array}{c}1.153(0.272-5.069) \\
\text { vs. } 5.182(0.044-102.491) \\
0.049\end{array}$ \\
\hline TSC2 & 0.03 & $\begin{array}{c}0.537(0.083-15.495) \\
\text { vs } 0.858(0.168-1.301) \\
0.06\end{array}$ & $\begin{array}{c}0.537(0.083-15.495) \\
\text { vs. } 0.637(0.146-2.615) \\
0.026\end{array}$ & $\begin{array}{c}0.858(0.168-1.301) \\
\text { vs. } 0.637(0.146-2.615) \\
0.57\end{array}$ \\
\hline KRAS & 0.59 & $\begin{array}{c}2.677(0.381-623.826) \\
\text { vs. } 2.568(1.444-5.431) \\
0.61\end{array}$ & $\begin{array}{c}2.677(0.381-623.826) \\
\text { vs. } 3.653(0.047-27.306) \\
0.553\end{array}$ & $\begin{array}{c}2.568(1.444-5.431) \\
\text { vs. } 3.653(0.047-27.306) \\
0.27\end{array}$ \\
\hline$S 6 K 1$ & 0.0045 & $\begin{array}{c}0.563(0.019-250.998) \\
\text { vs. } 0.913(0.335-3.641) \\
0.14\end{array}$ & $\begin{array}{c}0.563(0.019-250.998) \\
\text { vs. } 3.967(0.069-55.040) \\
0.003\end{array}$ & $\begin{array}{c}0.913(0.335-3.641) \\
\text { vs. } 3.967(0.069-55.040) \\
0.123\end{array}$ \\
\hline TP53 & 0.0003 & $\begin{array}{c}0.707(0.004-63.554) \\
\text { vs. } 1.256(0.300-2.249) \\
0.03\end{array}$ & $\begin{array}{c}0.707(0.004-63.554) \\
\text { vs. } 1.357(0.194-7.797) \\
0.0003\end{array}$ & $\begin{array}{c}1.256(0.300-2.249) \\
\text { vs. } 1.357(0.194-7.797) \\
0.55\end{array}$ \\
\hline EIF4E & 0.025 & $\begin{array}{c}0.708(0.071-2633) \\
\text { vs. } 0.772(0.406-3.096) \\
0.68\end{array}$ & $\begin{array}{c}0.708(0.071-2633) \\
\text { vs. } 5.422(0.124-102.554) \\
0.014\end{array}$ & $\begin{array}{c}0.772(0.406-3.096) \\
\text { vs. } 5.422(0.124-102.554) \\
0.04\end{array}$ \\
\hline$L K B 1$ & 0.0001 & $\begin{array}{c}0.508(0.014-58.432) \\
\text { vs. } 0.736(0.340-1.712) \\
0.054\end{array}$ & $\begin{array}{c}0.508(0.014-58.432) \\
\text { vs. } 1.348(0.156-11.753) \\
0.0001\end{array}$ & $\begin{array}{c}0.736(0.340-1.712) \\
\text { vs. } 1.348(0.156-11.753) \\
0.07\end{array}$ \\
\hline PIK $3 C A$ & $<0.0001$ & $\begin{array}{c}0.388(0.038-63.337) \\
\text { vs. } 1.112(0.141-3.256) \\
0.018\end{array}$ & $\begin{array}{c}0.388(0.038-63.337) \\
\text { vs. } 0.998(0.135-4.598) \\
<0.0001\end{array}$ & $\begin{array}{c}1.112(0.141-3.256) \\
\text { vs. } 0.998(0.135-4.598) \\
0.98\end{array}$ \\
\hline BECN1 & 0.0014 & $\begin{array}{c}0.599(0.018-9.815) \\
\text { vs. } 1.077(0.452-2.245) \\
0.019\end{array}$ & $\begin{array}{c}0.599(0.018-9.815) \\
\text { vs. } 1.197(0.161-8.006) \\
0.002\end{array}$ & $\begin{array}{c}1.077(0.452-2.245) \\
\text { vs. } 1.197(0.161-8.006) \\
0.65\end{array}$ \\
\hline $4 E B P 1$ & 0.027 & $\begin{array}{c}0.488(0.062-97.736) \\
\text { vs. } 0.799(0.330-5.507) \\
0.14\end{array}$ & $\begin{array}{c}0.488(0.062-97.736) \\
\text { vs. } 1.574(0.225-73.041) \\
0.015\end{array}$ & $\begin{array}{c}0.799(0.330-5.507) \\
\text { vs. } 1.574(0.225-73.041) \\
0.49\end{array}$ \\
\hline$A K T 1$ & 0.16 & $\begin{array}{c}0.623(0.025-244.898) \\
\text { vs. } 0.952(0.412-5.140) \\
0.48\end{array}$ & $\begin{array}{c}0.623(0.025-244.898) \\
\text { vs. } 1.690(0.193-99.022) \\
0.073\end{array}$ & $\begin{array}{c}0.952(0.412-5.140) \\
\text { vs. } 1.690(0.193-99.022) \\
0.31\end{array}$ \\
\hline
\end{tabular}

*Kruskal-Wallis test p-value (statistically significant value of less than 0.05 ) for comparison of three groups; **Relative quantity (RQ) median values (minimum-maximum); ***Wilcoxon test p-value (statistically significant value of less than 0.05 )

The decrease of autophagic capacity, related to lower beclin-1 expression, has been recently shown by immunohistochemical studies in epithelial ovarian cancer [18]. It has been found that $B E C N 1$ gene levels were significantly higher in benign and borderline ovarian tumors than in epithelial ovarian carcinoma. In another study, higher expressions of Beclin-1 protein were found in normal and benign ova- 
A

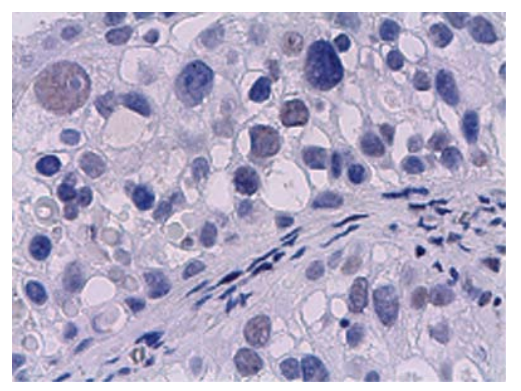

D

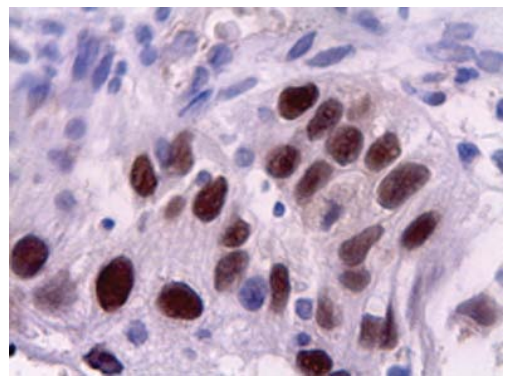

G

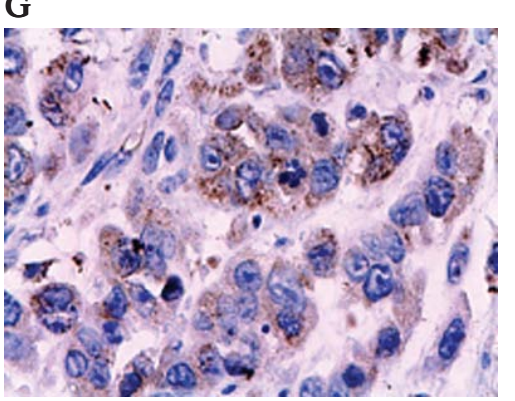

$\mathbf{J}$

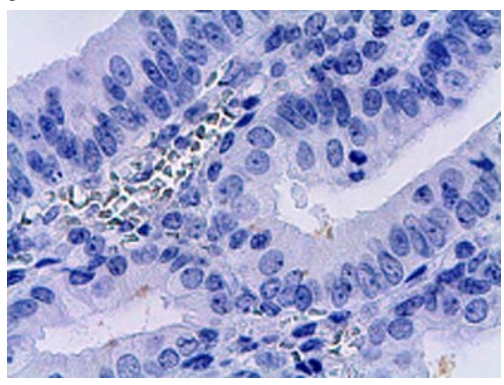

B

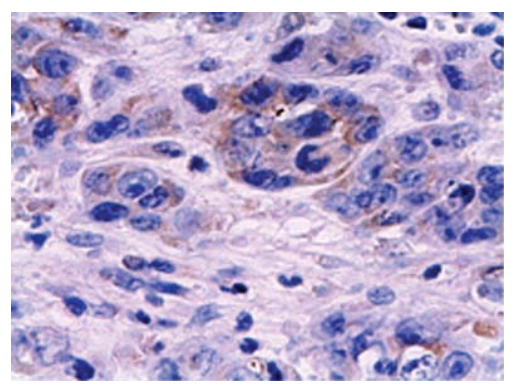

$\mathrm{E}$

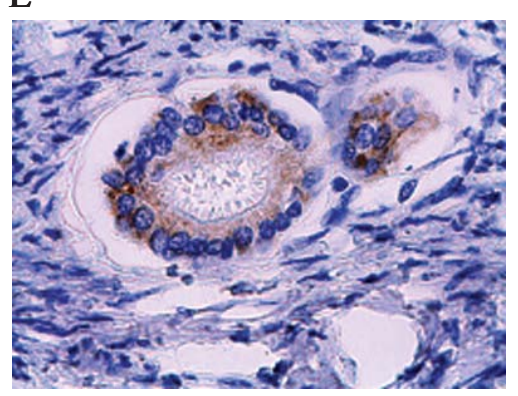

$\mathbf{H}$

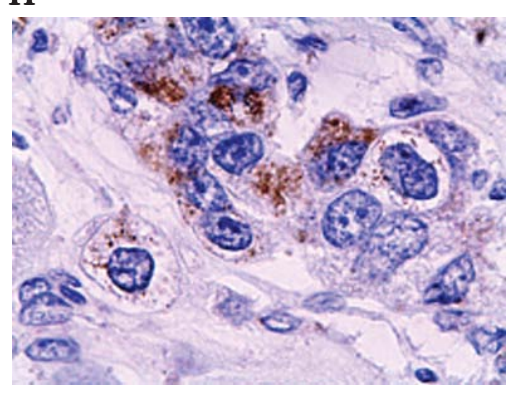

C

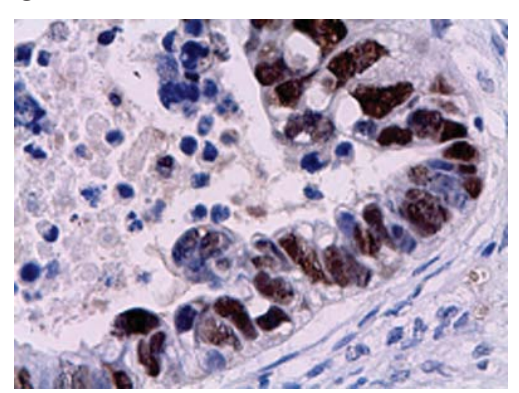

F

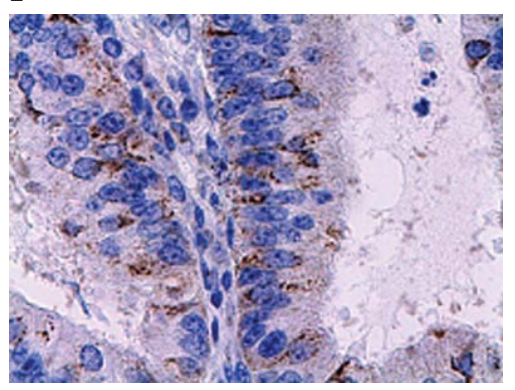

I

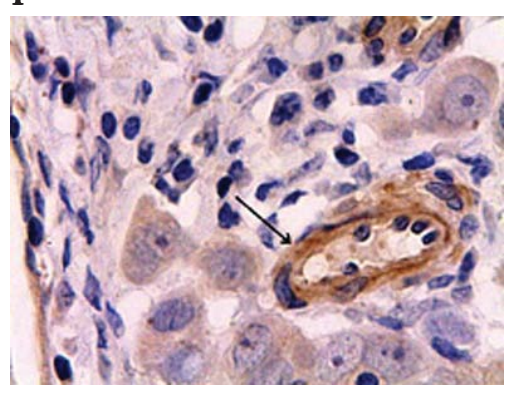

Figure 1. Immunolocalization of PTEN, TP53, PIK3CA and BECN1 in ovarian cancer. Photos show (magnification $\times 400$ ) selected histological sections of four main types i.e. serous, endometrioid, clear cell and mucinous. On (A) and (B) nuclear and cytoplasmic expression of PTEN is presented in clear cell and mucinous types of ovarian cancer respectively; (C) and (D) show nuclear TP53 localization in endometrioid and clear cell types; PIK3CA cytoplasmic reaction is shown in $(\mathbf{E}),(\mathbf{F}),(\mathbf{G})$ and $(\mathbf{H})$, respectively in: serous, endometrioid, mucinous and clear cell types; (I) and (J) show weak cytoplasmic BECN1 staining, respectively in serous and endometrioid cancer cells. Arrows in (I) indicate strong BECN1 endothelial expression in serous ovarian cancer

rian neoplasia tissues; the expressions were reduced in the borderline lesion tissue and the lowest level of expressions could be detected in the ovarian carcinoma tissue [19]. Although two of these studies were published in Chinese, making it difficult to describe methodology, we believe that the immunostaining of Beclin-1 was prevalent in ovarian cancer specimens. In our study, we for the first time confirmed that the median mRNA expression level is lower in malignant compared to non-malignant lesions of the ovaries, but the protein immunolocalization is relatively scarce and weak.

Decreased levels of TP53 are not surprising since inactivation of this gene appears to be an early step in ovarian carcinogenesis and results most commonly from mutations, but also from export from nuclei mutations in proteins regulating p53 activity, or overexpression of MDM2 - an important negative regulator of the TP53[20]. On the other hand, some interesting new studies have recently been published on the role of PIK3CA in ovarian cancer, which is frequently amplified, as well as its expression increased, at the RNA and protein level [21]. Approximately $40 \%$ of ovarian cancers show increased copy numbers at $3 \mathrm{q} 26$, which contains PIK3CA [21, 22]. Interestingly, the frequency of PI3K-increases, and the RNA and protein levels exceed those at the DNA level, suggesting that copy 
number-independent mechanisms also regulate $P I 3 K$ levels in ovarian cancer.

Although most studies have found increased amplification and mRNA expression, as cited above, it was recently shown that the expression level of PIK3CA does not differ between ovarian cancer and healthy ovaries. In order to study the expression profile of PI3K family in ovarian cancer, Zhang et al. retrieved from their website [23] public expression microarray data sets of human cancer and analyzed data by a web-based microarray bioinformatic tool called Oncomine [24]. After analysis of nine independent microarray studies, including two ovarian cancer ones, they found that out of six significantly amplified PI3K genes, including PIK3CA, only PIK3R3 had a significantly upregulated mRNA expression level in ovarian cancer compared to normal ovary in both ovarian cancer studies [23]. No difference as to the expression of PIK3CA was observed.

The differences as to the expression of PIK3CA between other studies and our own may be partly due to efficiency and adequacy in the collection of ovarian, malignant and non-malignant specimens (most of the articles overlook this, or do not include detailed data of RNA integrity analysis). In our study, after RNA isolation and assessment of its integrity (by means of so called RIN values - see methods) we found that only in 53 of the initial 80 patients with ovarian cancer were we able to obtain RNA that fulfilled the acceptable criteria of RIN of at least 7 with relatively clearly visible bands [25]. On the other hand, the median RIN values of healthy ovaries (successful collection in 11 of 19 patients), were about 8 , and the bands were in every case particularly clear (data not shown). This in our view might point to lesser degradation of this type of sample which could result in higher absolute levels of expression of some of the genes, possibly PIK3CA.

It is important to keep in mind that this may not necessarily point to their potential role in this type of tissue, since expression of three tumor suppressor genes were, as could be expected, significantly lower. This could suggest lesser degradation of corresponding mRNAs. To be more certain of the most proper tissue selection, laser captured microdissection is recommended which allows for targeted isolation of RNA from clearly defined types of cells. This technique is currently being introduced in our laboratory in order to assure the best sample selection.

In summary, our study shows that the low-density pathway-focused qualitative gene array is a robust and reproducible technique for quantifying expression of multiple genes in such a heterogenous sample as the ovary, including malignant and non-malignant lesions. Our results also show that the downregulation of some tumor suppressor genes and oncogenes may be associated with the pathogenesis of ovarian cancer.

\section{Acknowledgements}

This study was supported by Grant number KBN 2 PO5E 03629 from the State Committee for Scientific Investigation.

\section{Conflict of interest}

The authors declare that there are no conflicts of interest.

\section{References}

1. Society AC. Cancer facts \& figures. Atlanta, GA: The Society. 2007.

2. Sabatini DM. mTOR and cancer: insights into a complex relationship. Nature Reviews Cancer. 2006;6:729-734.

3. Laudanski P, Szamatowicz J, Kowalczuk O, Kuzmicki M, Garbowicz M, Chyczewski L. Expression of selected tumor suppressor and oncogenes in endometrium of women with endometriosis. Hum Reprod. 2009;24:1880-1890.

4. Castellvi J, Garcia A, Rojo F et al. Phosphorylated 4E binding protein 1: a hallmark of cell signaling that correlates with survival in ovarian cancer. Cancer. 2006;107: 1801-1811.

5. Vivanco I and Sawyers CL. The phosphatidylinositol 3-kinase AKT pathway in human cancer. Nat Rev Cancer. 2002; 2:489-501.

6. Sansal I and Sellers WR. The biology and clinical relevance of the PTEN tumor suppressor pathway. J Clin Oncol. 2004;15:2954-2963.

7. Kwiatkowski DJ. Tuberous sclerosis: from tubers to mTOR. Ann Hum Genet. 2003;67:87-96.

8. Basso AD, Mirza A, Liu G, Long BJ, Bishop WR, Kirschmeier P. The farnesyl transferase inhibitor (FTI) SCH66336 (lonafarnib) inhibits Rheb farnesylation and mTOR signaling. Role in FTI enhancement of taxane and tamoxifen anti-tumor activity. J Biol Chem. 2005;280:31101-31108.

9. Shaw RJ and Cantley LC. Ras, PI(3)K and mTOR signalling controls tumour cell growth Nature. 2006;441:424-430.

10. Vogelstein B, Lane D and Levine AJ. Surfing the p53 network. Nature. 2000;408:307-310.

11. Jin S and White E. Role of autophagy in cancer: management of metabolic stress. Autophagy. 2007;3:28-31.

12. Kamada Y, Funakoshi T, Shintani T, Nagano K, Ohsumi M and Ohsumi Y. Tor-mediated induction of autophagy via an Apg1 protein kinase complex. J Cell Biol. 2000;150:1507-1513.

13. Wullschleger S, Loewith $\mathrm{R}$ and Hall MN. TOR signaling in growth and metabolism. Cell. 2006;124:471-484.

14. Livak KJ and Schmittgen TD. Analysis of relative gene expression data using real-time quantitative PCR and the 2(-Delta Delta C(T)) Method. Methods. 2001;25 402-408.

15. Mutter GL. Pten, a protean tumor suppressor. Am J Pathol. 2001;158:1895-1898.

16. Schondorf T, Hoopmann M, Eversheim B et al. Expression of the tumor suppressor gene PTEN is not altered in the progression of ovarian carcinomas and does not correlate with p27Kip1 expression. Oncol Rep. 2003;10:1717-1722.

17. Chen Y, Zheng H, Yang X, Sun L and Xin Y. Effects of mutation and expression of PTEN gene mRNA on tumorigene- 
sis and progression of epithelial ovarian cancer. Chin Med Sci J. 2004;19:25-30.

18. Shen Y, Liang LZ, Hong MH, Xiong Y, Wei M, Zhu XF. Expression and clinical significance of microtubule-associated protein 1 light chain 3 (LC3) and Beclin1 in epithelial ovarian cancer. Ai Zheng. 2008;27:595-599.

19. Duan ZL, Peng ZL, Wang ZH, Yan NH. Correlation of autophagy gene Beclin1 to tumorigenesis and development of epithelial ovarian cancer. Ai Zheng. 2007;26:258-263.

20. Corney DC, Flesken-Nikitin A, Choi J, Nikitin AY. Role of p53 and Rb in ovarian cancer. Adv Exp Med Biol. 2008; 622:99-117.

21. Shayesteh L, Lu Y, Kuo WL et al. PIK3CA is implicated as an oncogene in ovarian cancer. Nat Genet. 1999;21:99-102.
22. Suzuki S, Moore DH $2^{\text {nd }}$, Ginzinger DG et al. An approach to analysis of large-scale correlations between genome changes and clinical endpoints in ovarian cancer. Cancer Res. 2000;60:5382-5385.

23. Zhang L, Huang J, Yang $\mathrm{N}$ et al. Integrative genomic analysis of phosphatidylinositol 3'-kinase family identifies PIK3R3 as a potential therapeutic target in epithelial ovarian cancer. Clin Cancer Res. 2007;13:5314-5321.

24. Rhodes DR, Yu J, Shanker K et al. ONCOMINE: a cancer microarray database and integrated data-mining platform. Neoplasia. 2004;6:1-6.

25. Schroeder A, Mueller O, Stocker S et al. The RIN: an RNA integrity number for assigning integrity values to RNA measurements. BMC Mol Biol. 2006;7:3.

Submitted: 4 December, 2010 Accepted after reviews: 30 January, 2011 